University of Warwick institutional repository: http://go.warwick.ac.uk/wrap This paper is made available online in accordance with publisher policies. Please scroll down to view the document itself. Please refer to the repository record for this item and our policy information available from the repository home page for further information.

To see the final version of this paper please visit the publisher's website. Access to the published version may require a subscription.

Author(s): J. R. Barker, M. Mudares, B. R. Snell, P. S. S. Guimaraes, D. C. Taylor, L. Eaves, and G. Hill Article Title: Validation of magnetophonon spectroscopy as a tool for analyzing hot-electron effects in devices

Year of publication: 1985

Link to published version: http://dx.doi.org/10.1063/1.96175

Publisher statement: none 


\title{
Validation of magnetophonon spectroscopy as a tool for analyzing hot- electron effects in devices
}

\author{
J. R. Barker and M. Mudares \\ Department of Physics, University of Warwick, Coventry CV4 7AL, United Kingdom \\ B. R. Snell, P. S. S. Guimaraes, D. C. Taylor, and L. Eaves \\ Department of Physics, University of Nottingham, Nottingham, United Kingdom \\ G. Hili \\ Department of Electrical Engineering, University of Sheffield, Sheffield, United Kingdom
}

(Received 15 April 1985; accepted for publication 31 May 1985)

\begin{abstract}
It is shown that very high precision hot-electron magnetophonon experiments made on $n^{+} n^{-} n^{+}$GaAs sandwich device structures which are customized for magnetoresistance measurements can be very accurately modeled by a new Monte Carlo technique. The latter takes account of the Landau quantization and device architecture as well as material parameters. It is proposed that this combination of experiment and modeling yields a quantitative tool for the direct analysis of spatially localized very nonequilibrium electron distributions in small devices and low dimensional structures.
\end{abstract}

In this letter we present a series of longitudinal magnetoresistance measurements and Monte Carlo simulations on short ( $\approx$ few microns) $n^{+} n^{-} n^{+}$-GaAs mesa structures. Remarkably good agreement is obtained between the calculated and experimental data. The Monte Carlo method, which is a new precision variant of the ensemble method, accounts for both the magnetic field dependence of the monotonic background over a wide range of applied electric field and also of the weak, but well-resolved magnetophonon resonance structure superimposed upon this background. In particular, the observed electric field dependence of the amplitudes of the conventional and pseudoresonance magnetophonon peaks are accurately reproduced in the Monte Carlo simulation. Exceptionally high precision is required in both the experimental measurements and the simulation because the oscillatory components can be less than $0.1 \%$ of the total response. The complexity of the magnetophonon structure is well known ${ }^{1-4}$ and for the technique to be fully exploited it is crucial to have a precision theoretical interpretation of the data. For this purpose we have made a detailed test study of the hot-electron longitudinal magnetophonon effect in devices fabricated as $n^{+} n^{-} n^{+}$-GaAs sandwich structures with active layer thickness $5-9 \mu \mathrm{m}$ for $E$ up to $5 \mathrm{kV} / \mathrm{cm}$ and $B$ up to $12 \mathrm{~T}$ over the temperature range $77-300 \mathrm{~K}$. Although the magnetophonon oscillations were found to be extremely weak, less than $0.1 \%$ of the background, a large number of damped resonances were detectable including a hitherto unreported strong electric field dependent pseudoresonance structure close to the half-integer resonance conditions.

Magnetophonon resonance occurs at magnetic fields given by $N \hbar \omega_{c}=N \hbar e B / m^{*}=\hbar \omega_{L}$, where $\hbar \omega_{c}$ is the cyclotron energy, $m^{*}$ is the electronic effective mass, and $\hbar \omega_{L}$ is the energy of the longitudinal optical (LO) phonon mode at the center of the Brillouin zone. In the longitudinal configuration minima usually occur at the resonant fields given by the above expression. For ohmic conduction the minima arise from the competition between elastic and inelastic LO phonon emission processes in limiting the longitudinal carrier momentum. At higher electric fields the resonant structure becomes dominated by processes which derive from res- onant energy relaxation when carrier heating becomes important. ${ }^{3}$ In addition, auxiliary structures known as pseudoresonances are also observed in $\rho_{z z}$ at magnetic fields given by $2 \hbar \omega_{L}=M \hbar \omega_{c}(M=1,2,3, \ldots)$. The latter derive from an additional resonance which is determined by an interplay between LO phonon absorption and emission processes in limiting the longitudinal carrier momentum. ${ }^{2,3}$

The structures were in the form of $n^{+} n^{-} n^{+}$sandwiches prepared by liquid phase epitaxy. An active layer of lightly doped $\mathrm{GaAs}\left(1 \times 10^{15} \mathrm{~cm}^{-3}\right)$ was grown on the top of a 100 $\mu$ m-thick $n^{+}$substrate $\left(2 \times 10^{18} \mathrm{~cm}^{-3}\right)$. Samples with active layer thicknesses of 5 and $9 \mu \mathrm{m}$ were investigated. The top layer was $0.3 \mu \mathrm{m}$ thick with $n^{+}=2 \times 10^{17} \mathrm{~cm}^{-3}$. Photolithography was used to form mesas with a diameter of 0.1 and $0.2 \mathrm{~mm}$. The electrical contacts to the sample were made via $\mathrm{Au}-\mathrm{Ge}$ alloy layers.

For measurements of $\rho_{z z}$, the above geometry has considerable advantages over measurements involving current from the plane of an epitaxial layer. In particular, bending of current lines ${ }^{5}$ due to contacts and doping inhomogeneities is avoided as is pinch-off of the conduction channel by depletion at the surface and substrate interface.

The experiments were simulated using the ensemble Monte Carlo method as the basis for full device modeling which took into account Poisson corrections for the local electric field distribution throughout all the layers of the device. This procedure is essential to establish the nonlinear relation between the local voltage distribution in the device and the applied bias. Separate calculations based on bulk boundary conditions were found to be inadequate and led to approximately $10 \%$ errors in the background magnetoresistance. The ensemble technique used 75000 test particles for each simulation where the intercollisional dynamics and scattering rates were computed for collision-damped Landau states for electrons in a three-valley $(\Gamma-L-X$ ordered) nonparabolic model of GaAs. We believe that this is the first occasion on which Monte Carlo calculations have been performed for magnetically quantized states. It was found sufficient to evaluate the electron distribution among up to 10 Landau levels. The magnetic field dependent scattering rates 


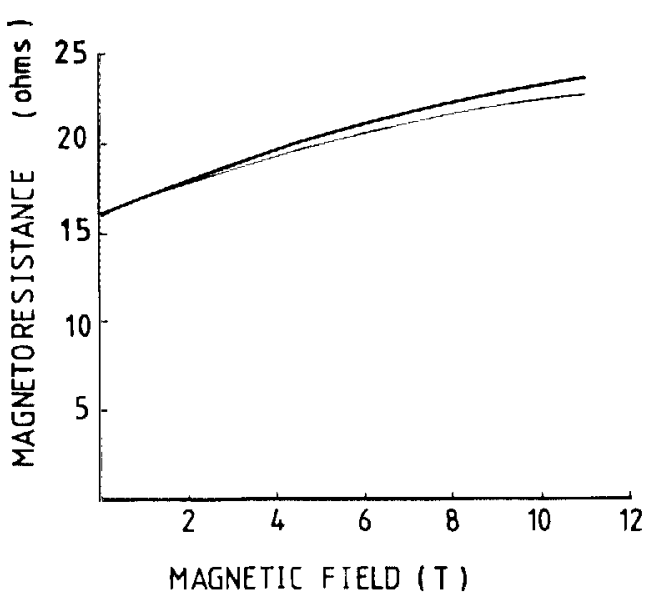

FIG. 1. Measured (thin curve) and theoretical (thick curve) magnetoresistance $(140 \mathrm{~K})$ of an $n^{+} n^{-} n^{+}$.GaAs sand wich structure for a constant current $10 \mathrm{~mA}$.

were evaluated for polar and nonpolar optical phonon scattering, elastic acoustic, and piezoelectric scattering, $\Gamma-L / \Gamma$ $X / L-L / X-X$ intervalley scattering, and ionized impurity scattering (material parameters, corrected to the appropriate temperatures, were based on Refs. 6 and 7). Collision damping was assumed to be approximated by a Lorentzian spectral density function with a damping factor $g=0.3$. Very high convergence was achieved by deploying an iterative scheme in which the starting electron distribution for each successively incremented magnetic field strength is selected as the steady-state distribution evaluated at the previous magnetic field value.

The experiments were performed in a constant current mode so that the voltage drop across the sample is proportional to the magnetoresistance. The Monte Carlo simulation of the constant current mode was obtained by a decon-

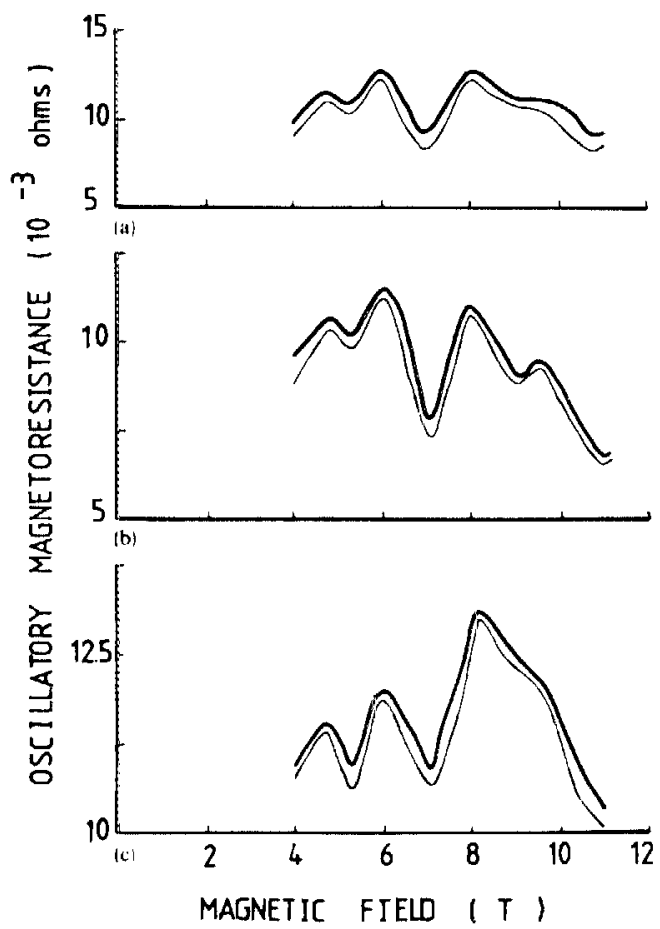

FIG. 2. Measured (thin curve) and theoretical (thick curve) oscillatory component of magnetoresistance as a function of applied bias. (a) Constant current $10 \mathrm{~mA}$; (b) constant current $20 \mathrm{~mA}$; (c) constant current $40 \mathrm{~mA}$.

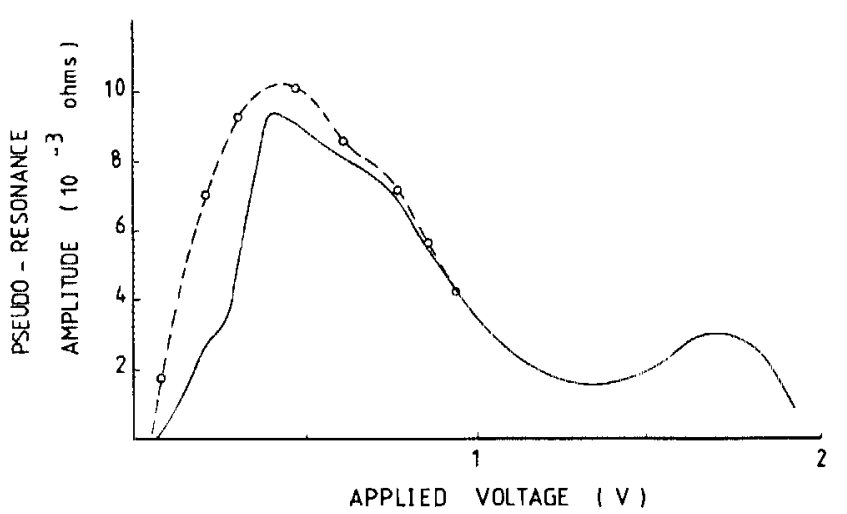

FIG. 3. Amplitude dependence of the $M=5$ pseudoresonance at $140 \mathrm{~K}$ as a function of applied voltage. (O) Experiment; (full curve) theory.

volution from ensemble calculations based on a constant voltage mode. Figures 1 and 2 compare the theory and experiment over a representative range of current values which correspond to weak [Figs. 1 and 2(a)], intermediate [Fig. 2(b)], and high [Fig. 2(c)] electric fields. As can be seen from Fig. 1 the agreement with the overall magnetoresistance $R(B)$ is very good. Inspection of the figure also shows that the oscillatory component is extremely weak $(\leqslant 0.1 \%)$. The oscillatory structure is revealed in Figs. $2(\mathrm{a})-2(\mathrm{c})$ by subtracting from $R(B)$ a cubic fitting function $F_{3}(B)$. It must be stressed that the same $F_{3}(B)$ is used to extract the oscillations in both the simulated and experimental magnetoresistance. Again, the agreement between theory and experiment is very good for both the overall amplitudes and the harmonic content of the oscillations.

A particularly interesting feature of the results is the observation of the strong electric field dependence of the $M=5$ pseudoresonance magnetoresistance minimum at about $B=9 \mathrm{~T}$. The amplitude dependence of this feature on applied voltage (and electric field) is illustrated in Fig. 3. The observed rise and fall of the amplitude which are accurately simulated by the Monte Carlo method may be qualitatively explained as follows. The decay of the amplitude at high field is associated with the buildup of strong streaming of the electron distributions in each Landau level which moves carriers away from the pseudoresonance condition. This result is consistent with an earlier and less substantial rate equation calculation. ${ }^{3,8}$ In addition, the relative weakening of $\mathrm{LO}$ phonon absorption compared to LO phonon emission processes also contributes to this effect. The rise in amplitude at weak to intermediate electric fields is consistent with the rise in the electron temperature (correlated with the symmetric part of the electron distribution) which may be understood. on the basis of the elementary ohmic theory described elsewhere. ${ }^{2.4}$

To summarize, this letter shows that high precision experiments on device structures which are customized for magnetoresistance measurements can be very accurately simulated by Monte Carlo methods which take account of both the material parameters and the architecture of the device. Even in these relatively simple structures the interaction between the $n^{+}$contact regions and the active layer plays a significant role in the interpretation of the device response to applied voltages and magnetic fields. This validation of magnetophonon spectroscopy as a quantitative tool 
for analyzing hot-electron effects offers hope that the techniques may be used to directly measure the spatially localized, very nonequilibrium processes which occur in submicron devices. It should be possible, for example, to obtain resonant energy relaxation via surface and interfacial optical phonons, thus providing a direct probe of both the interfaces and the electron distributions.

This work is partly supported by the Science and Engineering Research Council.
'P. G. Harper, J. W. Hodby, and R. A. Stradling, Rep. Prog. Phys. 36, 1 (1973).

${ }^{2}$ R. L. Peterson, Semiconductors and Semimetals 10, 221 (1975).

${ }^{3}$ J. R. Barker, Solid State Electron. 21, 197 (1978).

${ }^{4}$ J. R. Barker, in Handbook on Semiconductors, edited by W. Paul (NorthHolland, Amsterdam, 1982), Vol. 1, Chap. 11C, p. 617

${ }^{5}$ L. Eaves, Ph.D. thesis, Oxford University 1972.

${ }^{6}$ M. A. Littlejohn, J. R. Hauser, and T. H. Glisson, J. Appl. Phys. 48, 4587 (1977).

${ }^{7}$ N. M. Ravindra and V. K. Srivastava, J. Phys. Chem. Solids 40, 791 (1979).

${ }^{8}$ J. R. Barker and B. L. Magnusson, Phys. Lett. A 54, 283 (1975).

\title{
Laser-induced photochemical etching of $\mathrm{SiO}_{2}$ studiled by $x$-ray photoelectron spectroscopy
}

\author{
S. Yokoyama, Y. Yamakage, and M. Hirose \\ Department of Electrical Engineering, Hiroshima University, Higashihiroshima 724, Japan
}

(Received 8 February 1985; accepted for publication 3 June 1985)

\begin{abstract}
Thermally grown $\mathrm{SiO}_{2}$ on $\mathrm{Si}$ and quartz wafers have been photochemically etched in an $\mathrm{NF}_{3}$ gas containing $0-4$ mol \% hydrogen by using an ArF excimer laser (193 nm in wavelength) irradiation. In situ $\mathrm{x}$-ray photoelectron spectroscopy of the etched surface at each step of the photochemical reaction and in situ infrared absorption measurements of the etching gas have revealed the elementary process of etching reactions on the surface and the resulting products in the gas phase.
\end{abstract}

Photochemical reaction inherently induces smaller damage on the solid surface than plasma reaction in which the high-energy ion bombardment tends to cause the surface damage. Since laser beam can be easily focused in small dimensions and also can be projected onto the surface through a mask pattern, selective photochemical deposition, etching, and doping can be accomplished. These features of photochemical processes are potentially important for the future process technology to fabricate very large scale integrated (VISI) circuits. Laser-induced etching of $\mathrm{SiO}_{2}$ has been attempted by Steinfeld et al. ${ }^{\prime}$ in $\mathrm{CF}_{3} \mathrm{Br}$ by $\mathrm{CO}_{2}$ laser irradiation. In this case $\mathrm{CF}_{3} \mathrm{Br}$ was dissociated by vibrational excitation and the created radicals react with $\mathrm{SiO}_{2}$. Houle and Chuang ${ }^{2}$ have also achieved $\mathrm{SiO}_{2}$ etching in $\mathrm{Cl}_{2}$ by Ar laser irradiation, and they claimed that thermal reaction rather than pure photochemical reaction seems to be dominant.

In this letter the kinetics of photochemical etching reactions of $\mathrm{SiO}_{2}$ in an $\mathrm{NF}_{3}+\mathrm{H}_{2}$ gas mixture by $\mathrm{ArF}$ excimer laser irradiation has been studied by in situ $\mathrm{x}$-ray photoelectron spectroscopy (XPS) together with infrared (IR) absorption measurements of the etching gas.

An ArF excimer laser light (193 $\mathrm{nm}$ in wavelength) with a pulse energy of $\sim 7.7 \mathrm{~mJ} /\left(\mathrm{cm}^{2}\right.$ shot) from Lambda Physik EMG $103 \mathrm{E}$ was irradiated at a repetition rate of $80 \mathrm{~Hz}$ onto $\mathrm{Si}$ wafers covered with thermally grown $\mathrm{SiO}_{2}$ with thicknesses of $10-300 \mathrm{~nm}$ or onto quartz sheets $10.3 \mathrm{~mm}$ in thickness) in $\mathrm{NF}_{3}$ containing $0-4 \mathrm{~mol} \%$ hydrogen. The temperature rise of the Si substrate due to laser irradiation is estimated to be less than $40^{\circ} \mathrm{C} .{ }^{3} \mathrm{NF}_{3}$ gas has currently been used in the plasma etching of $\mathrm{Si}$ and $\mathrm{SiO}_{2}$, and a mixture of $\mathrm{H}_{2}$ gas with $\mathrm{CF}_{4}$ has also been employed for improving the etching selectivity of $\mathrm{SiO}_{2}$ with respect to $\mathrm{Si}^{4,5}$ The absorption coefficient of $\mathrm{NF}_{3}$ gas was measured in advance to determine the optical absorption cross section at 193-nm irradiation. The result yielded a cross section of $5.3 \times 10^{-21} \mathrm{~cm}^{2}$. The oxide thickness on the silicon substrate at each step of etching was measured by the infrared absorption intensity of the $\mathrm{Si}-\mathrm{O}$ stretching mode at $1060 \mathrm{~cm}^{-1}$ as well as by the multibeam interferometry. Etched pattern of $\mathrm{SiO}_{2}$ was directly observed by scanning electron micrograph (SEM). The $\mathrm{x}$-ray photoelectron spectra of the phototreated $\mathrm{SiO}_{2}$ surfaces were measured using a Shimadzu ESCA750H spectrometer in which the specimen can be transferred from the reaction chamber to the ultrahigh vacuum XPS chamber through the two gate valves, and the chemical bonding feature of the $\mathrm{SiO}_{2}$ surface was analyzed. A detailed description of the XPS system is given elsewhere. ${ }^{6}$ The in situ infrared absorption spectra of the etching gas in the reaction chamber were also measured to identify the etching procucts in the gas phase. The etching rate of $\mathrm{SiO}_{2}$ was measured for various configurations between the incident laser beam and the $\mathrm{SiO}_{2}$ surface. It is found that when the $\mathrm{SiO}_{2}$ surface is photoprocessed for $60 \mathrm{~s}$ in $\mathrm{NF}_{3}+\mathrm{H}_{2}$ at a low pressure $\left\langle\mathrm{NF}_{3}=480 \mathrm{~Pa}\right.$, $\mathrm{H}_{2}=20 \mathrm{~Pa}$ ), followed by photoprocessing at an atmospheric pressure $\left(\mathrm{NF}_{3}=9.8 \times 10^{4} \mathrm{~Pa}, \mathrm{H}_{2}=2.6 \times 10^{3} \mathrm{~Pa}\right)$, the photochemical etching reaction of $\mathrm{SiO}_{2}$ promptly starts to proceed, while without preirradiation at low pressure the incubation time of more than $120 \mathrm{~s}$ is necessary for initiating the 